\title{
De Novo Design of a Cytochrome $b$ Maquette for Electron Transfer and Coupled Reactions on Electrodes
}

\author{
Xiaoxi Chen, ${ }^{\dagger}$ Bohdana M. Discher, ${ }^{\ddagger}$ Denis L. Pilloud, ${ }^{\ddagger}$ Brian R. Gibney, \\ Christopher C. Moser, ${ }^{*}$ and P. Leslie Dutton*,* \\ Johnson Research Foundation, Department of Biochemistry and Biophysics, University of Pennsylvania, \\ Philadelphia, Pennsylvania 19104, and Phylos Inc., 128 Spring St., Lexington, Massachusetts 02421
}

Received: June 8, 2001; In Final Form: September 26, 2001

\begin{abstract}
Experimental explorations of functional mechanisms in natural electron-transfer proteins are often frustrated by their fragility and extreme complexity. We have designed and synthesized four- $\alpha$-helix-bundle redox proteins, maquettes, that are much simplified and more robust than natural redox proteins and can be designed to bind onto electrode surfaces to facilitate systematic investigations. The points of interest that can be now assessed are not only the processes that govern biological assembly of equilibrium structures, electrochemistry, and electron tunneling rates but also how these factors are coupled together to effect redox driven catalysis. Here we describe maquettes that bis-histidine ligate protoporphyrin IX (heme), much like native $b$ cytochromes, as well as contain charged surface patches, much like native cytochrome $c$. The positively charged residues aid adsorption to negatively charged surfaces, such as gold electrodes modified by 11-mercaptoundecanoic acid, and facilitate cyclic voltammetry $(\mathrm{CV})$ measurements. CV demonstrates the reversible electrochemistry typical for cytochrome $b$ as well as the coupling of the $b$-heme oxidation and reduction to proton exchange. The $\mathrm{pH}$ dependency of redox midpoint potentials reveals a major (three $\mathrm{pH}$ units) shift of the $\mathrm{p} K_{\mathrm{a}}$ which matches the shift previously shown to originate in nearby glutamates ${ }^{1}$. The redox potentials correspondingly shift from $-0.24\left(\mathrm{pH}>\mathrm{p} K_{\text {red }}\right.$, deprotonated) to $-0.11 \mathrm{~V}$ ( $\mathrm{pH}<\mathrm{p} K_{\mathrm{ox}}$, protonated). The rate of electron transfer at zero driving force between the hemes and the gold electrode was determined to be $120 \mathrm{~s}^{-1}$, a rate consistent with tunneling through the mercaptoundecanoic acid spacer and suggesting that the coupled proton exchange is not rate limiting. Reduction of the heme in the presence of CO-saturated buffer shifted the oxidation peak from -0.2 to $+0.35 \mathrm{~V}$, indicating massive preferential $\mathrm{CO}$ binding to the reduced heme. Consistent with solution spectroscopy, $\mathrm{CO}$ must displace one axial histidine to the heme to form the His $-\mathrm{CO}$ form of the ferrous heme. The $\mathrm{CO}$ is released upon heme oxidation at high potentials. In contrast to coupled proton exchange, $\mathrm{CO}$ binding/release and ligand exchange are slow on the time scale of electron tunneling between the heme edge and the electrode.
\end{abstract}

\section{Introduction}

An alternative approach to study functional mechanisms in natural electron-transfer proteins ${ }^{2-5}$ employs de novo design and synthesis of proteins. ${ }^{6-8}$ This approach aims to minimize structural complexity with a view to maximize visualization of a designed function that is so often obscured in natural proteins. We are using these simplified synthetic protein maquettes ${ }^{9}$ to identify the requirements for protein assembly and incorporation of electron-transfer cofactors and to determine what controls in situ electrochemistry, kinetic activity, and the nature of coupled chemistry. The goal is to describe the natural engineering and mechanisms underlying specific functions in the more complex natural protein counterparts.

Earlier feasibility studies have demonstrated that four- $\alpha$-helixbundle heme protein maquettes can be designed and synthesized to display the characteristic in situ electrochemical and optical properties of typical hemes in natural $b$-type cytochromes. ${ }^{1,9-13}$ The great freedom of de novo design has also been exploited by creating novel patterns of protein surface residues to enhance

\footnotetext{
* Corresponding author. Fax: (215) 573-2235. E-mail: dutton@ mail.med.upenn.edu.

† University of Pennsylvania.

$\doteqdot$ Phylos Inc.
}

selected interactions between $\alpha$-helices and to drive assembly and organization on surfaces. ${ }^{14-16}$ The present work describes the engineering of a cytochrome $b$ maquette with a roughly dipolar surface charge distribution analogous to that found in the native water-soluble cytochrome $c$. A patch of net positive charge on cytochrome $c$ encourages the appropriate binding and orientation for electron transfer at corresponding negative patches on native redox protein partners.

This same charge orientation can also encourage the adsorption of protein monolayers on electrodes to facilitate the study of the electron-transfer properties. ${ }^{17}$ With protein monolayers adsorbed onto electrode surfaces, electrons can be injected and abstracted at will by regulating the potential of the electrode, and kinetic analysis is greatly simplified due to the absence of diffusional limitations on the rates. To prepare a protein monolayer on a gold electrode, the metal surface is usually modified with a monolayer of a linker molecule; the exposed functional group of the linker promotes assembly and immobilization of the protein on the surface while maintaining the native protein state. The deliberate chemical modification of gold surfaces using organosulfur compounds has developed into a convenient method for the preparation of molecular monolayers on electrodes and other surfaces. ${ }^{18}$ 
Prior work has established that self-assembled monolayers (SAMs) prepared with carboxyl-terminated alkanethiols (HS$\left.\left(\mathrm{CH}_{2}\right)_{n} \mathrm{COOH}\right)$ can serve as excellent gold surface modifiers for the immobilization of horse heart cytochrome $c$ (cyt $c$ ) in a stable electroactive state. ${ }^{19-21}$ In the present report, we use cyt $c$ immobilized on gold electrode surface coated with a monolayer of 11-mercaptoundecanoic acid (MUA) as a standard for comparison with de novo designed maquettes whose external charged amino acid distribution has been redesigned to enhance its interaction with a negatively charged surface.

The reduction and oxidation waves of the voltammograms of redox-center-containing proteins adsorbed on modified electrodes provide information not only on the redox potentials and surface coverage of the electroactive proteins, but also on the kinetics of electron transfer between the electrode metal and the redox centers in the adsorbed proteins. Electron-transfer rates between the electrode metal and the redox centers in the proteins can be calculated by simulating the potential-scan-rate-dependent voltammograms and comparing to the experimental data. ${ }^{17,22-25}$

In nature, electron transfer reactions are often coupled to other chemical events that are critical for physiological function and its regulation. For example, oxidation/reduction of redox cofactors in proteins is frequently coupled to proton binding/ release. This proton exchange is often a charge-compensating response by the protein to profound changes in the electrostatic potential caused by an internal redox reaction. ${ }^{26}$ Furthermore, redox-coupled proton exchange is organized in some natural proteins to effect vectorial transmembrane proton pumping. However, the mechanism is often obscure, as in the hemecontaining cytochrome oxidase. ${ }^{27,28}$ Cofactor redox state can also be coupled to other ligand binding/release, as in the high affinity of the poison $\mathrm{CO}$ for ferrous heme and low affinity to ferric heme observed in cytochrome oxidase, ${ }^{29}$ myoglobin, ${ }^{30}$ and cytochrome $c_{3} \cdot{ }^{31}$ Electrochemistry of immobilized heme protein maquettes on electrode provides a useful means to study electron-transfer coupled chemical events. Since the proteins are immobilized on the electrode, the potential can be controlled easily, electrons can be injected and removed from the hemes at will, and the $\mathrm{pH}$ and other chemical composition of the buffered electrolyte solution can be adjusted easily.

\section{Materials and Methods}

Peptide Sequences. The amino acid sequence of the helixloop-helix $\alpha_{1}-1-\alpha_{2}$ of the dimeric $\left(\alpha_{1}-1-\alpha_{2}-s-\right)_{2}$ protein maquette is

\section{$\mathrm{Ac}-\mathrm{KKG} \bullet \mathrm{CGG} \bullet \mathrm{KIWKLH} \bullet \mathrm{EQFLQQF} \bullet \mathrm{EQLLQLF} \bullet$ EQQLQQL•GGGGGG・QIWQLH•EQFLQQF• $\mathrm{EQLLQLF} \bullet \mathrm{EQQLKKL} \cdot \mathrm{KK}-\mathrm{CONH}_{2}$}

A pair of single-sequence $\alpha_{1}-1-\alpha_{2}$ 's are linked by a disulfide bond at their cysteinyl $\mathrm{N}$-termini to form a four- $\alpha$-helix-bundle $\left(\alpha_{1}-1-\alpha_{2}-s-\right)_{2}$, i.e., the cytochrome $b$ (cyt $\left.b\right)$ maquette, as shown in Figure 1A. The design of each $\alpha$-helix is based on a second generation maquette described by Gibney et al..$^{32}$ that has unique structure in the apo form. The charged residues in the sequence are modified from the prototype so that lysines are concentrated toward one end of the four- $\alpha$-helix bundle while glutamates and glutamines are distributed from the middle toward the other end of the bundle. The 16 lysines and 12 glutamates in each four- $\alpha$-helix bundle confer net positive charge on the bundle at neutral $\mathrm{pH}$.
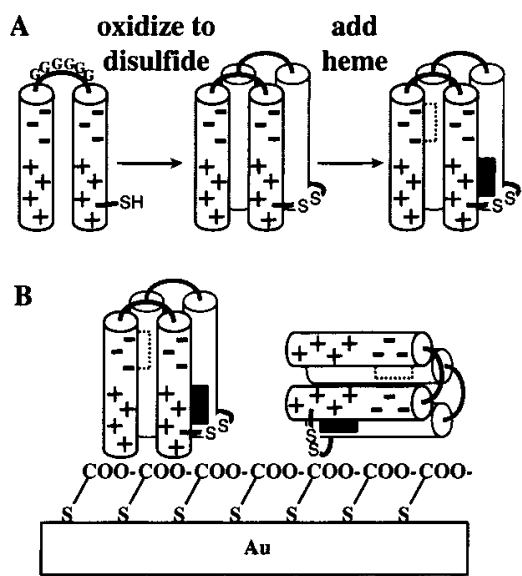

Figure 1. Design and construction of a cytochrome $b$ maquette. Part A: Solid-phase peptide synthesis produced the 68-amino acid peptide, $\alpha_{1}-1-\alpha_{2}$, with lysines (positively charged) concentrated on the opposite end of the glycine loop and glutamates (negatively charged) distributed in the middle region of the helices. $\alpha_{1}-1-\alpha_{2}$ is oxidized to form the disulfide bond and the 4-helix-bundle, $\left(\alpha_{1}-1-\alpha_{2}-s-\right)_{2}$. The 4-helixbundle can coordinate two hemes by bis-histidyl ligation to form the heme protein maquette, heme $-\left(\alpha_{1}-1-\alpha_{2}-s-\right)_{2}$. The first heme (black) binds tightly $\left(K_{\mathrm{d}}=17 \mathrm{nM}\right)$, the second heme (dashed line) binds weakly $\left(K_{\mathrm{d}}=3.5 \mu \mathrm{M}\right)$. The ratio of 0.7 heme/bundle assured that the cyt $b$ maquette does not contain more than one heme. Part B: Two possible orientation of the cyt $b$ maquette on the surface, with the helix axis perpendicular to the surface (cross section area of about $600 \AA^{2}$ / molecule) and with the helix axis parallel to the surface (cross section area of about $1200 \AA^{2} /$ molecule).

Chemicals and Solvents. Gold (99.99\%), titanium (99.99\%), hemin, trifluoroacetic acid (TFA), and dimethyl sulfoxide (DMSO) were purchased from Aldrich Chemical Co. (Milwaukee, WI). Cytochrome $c$ (from horse heart, 99\%) was purchased from Sigma Chemical Company (St. Louis, MO). Ethanedithiol and 1-hydroxy-benzotriazol were purchased from Fluka (Ronkonkoma, NY). Fmoc-protected amino acid perfluorophenyl esters were purchased from PerSeptive Biosystems (Framingham, MA). NovaSyn PR-500 resin was purchased from CalbiochemNovabiochem (La Jolla, CA). All chemicals and solvents were reagent grade. Water was purified using a Milli-Q water system from the Millipore Corp. (Bedford, MA).

Peptide Synthesis. The 68 -amino acid $\alpha_{1}-1-\alpha_{2}$ peptides were synthesized by a continuous-flow Milligen 9050 solidphase synthesizer using standard Fmoc/tBu protection strategy at $0.2 \mathrm{mmol}$ scale. The side-chain protecting groups used were as follows: Lys(tBoc), Glu(OtBu), and Cys(Trt). The peptides were cleaved from the resin and simultaneously deprotected using 90:8:2 trifluroacetic acid:ethanedithiol:water for $2 \mathrm{~h}$. Crude peptides were precipitated and triturated with cold ether, dissolved in water ( $0.1 \%$ TFA), lyophilized, and purified to homogeneity by reversed-phase C18 HPLC using aqueous acetonitrile gradients containing $0.1 \%(\mathrm{v} / \mathrm{v})$ TFA. The resulting peptide identities were confirmed with laser desorption mass spectrometry.

Protein Preparations. For cyclic voltammetry experiments, cyt $c$ was purified on a Sephadex G-25 M column (Column PD-10, Amersham Pharmacia Biotech) prior to protein adsorption. To prepare the cyt $b$ maquette, the 68-amino acid $\alpha_{1}-1-$ $\alpha_{2}$ peptides were dissolved in high ionic strength phosphate buffer $(50 \mathrm{mM}$ phosphate, $0.6 \mathrm{M} \mathrm{NaCl})$ at $\mathrm{pH} 8.0$ to yield a final concentration of $0.04 \mathrm{mM} \alpha_{1}-1-\alpha_{2}$. The peptide solution was then exposed to air to allow the cysteines to oxidize to form disulfide linked $\left(\alpha_{1}-1-\alpha_{2}-s-\right)_{2}$. Heme $-\left(\alpha_{1}-1-\alpha_{2}-s-\right)_{2}$ was formed by successive additions of 0.1 heme per binding 
site from a stock solution of $4 \mathrm{mM}$ of $\mathrm{Fe}(\mathrm{III})$ protoporphyrin IX (heme) in DMSO, until 1 heme per bis-histidine site was reached. During each addition, the solution was well stirred and then allowed to equilibrate for $5 \mathrm{~min}$. The final concentration of DMSO in the aqueous solution was always lower than 1:100 $(\mathrm{v}: \mathrm{v})$. The heme incorporation was monitored by the increase of the Soret band at $412 \mathrm{~nm}$. The heme binding properties were determined as described before. ${ }^{9,11}$ The measurements were performed at two different concentration $(0.215$ and $6 \mu \mathrm{M})$ and yielded two independent $K_{\mathrm{d}}$ values of $17 \mathrm{nM}$ and $3.5 \mu \mathrm{M}$. For the cyclic voltammetry and redox potentiometry measurements, the buffer composition was exchanged by Sephadex G-25 M column (Column PD-10, Amersham Pharmacia Biotech). This desalting procedure resulted in a loss of the weaker bound heme and therefore all experiments were performed at 0.7 heme/ bundle ratio.

Solution Molecular Weight Determination. Size-exclusion chromatography was performed on a Beckman System Gold HPLC system with a diode-array detector using a Supelco Sigmachrom GFC-100 column $(300 \times 7.5 \mathrm{~mm})$ eluted with aqueous buffer. The column was standardized using aprotinin (6.5 kDa), ribonuclease A (13.7 kDa), chymotrypsinogen (25.0 $\mathrm{kDa})$, ovalbumin $(43.0 \mathrm{kDa})$, and bovine serum albumin $(67.0$ $\mathrm{kDa}$ ).

Redox Potentiometry. Redox titrations were performed in an anaerobic cuvette equipped with a platinum working electrode and a saturated $\mathrm{KCl}$ calomel reference electrode. ${ }^{33}$ Ambient redox potentials (measured against the standard hydrogen electrode) were adjusted by aliquots of freshly prepared solutions of sodium dithionite or potassium ferricyanide. The titrations of cyt $c$ were performed in $50 \mathrm{mM}$ Tris, $100 \mathrm{mM} \mathrm{NaCl}$, and pH 7.0 and the titrations of cyt $b$ maquette in $60 \mathrm{mM} \mathrm{NaCl}, 5$ $\mathrm{mM}$ phosphate, and $\mathrm{pH}$ 8.0. Electrode-solution mediation was facilitated by redox mediators as follows: titrations of cyt $c$ were mediated by $40 \mu \mathrm{M}$ 2,3,5,6-tetramethyl- $p$-phenylenediamine, $20 \mu \mathrm{M}$ phenazine methosulfate, $20 \mu \mathrm{M}$ phenazine ethosulfate, $20 \mu \mathrm{M}$ 1,2-naphthoquinone, $20 \mu \mathrm{M}$ duroquinone, and $20 \mu \mathrm{M}$ 2-hydroxy-1,4-naphthoquinone; titrations of the cyt $b$ maquette were mediated by $5 \mu \mathrm{M}$ 1,2-naphthoquinone, $5 \mu \mathrm{M}$ 1,4-naphthoquinone, $20 \mu \mathrm{M}$ duroquinone, $10 \mu \mathrm{M}$ pyocyanine, $5 \mu \mathrm{M}$ indigotrisulfonate, $10 \mu \mathrm{M}$ 2-hydroxy-1,4-naphthoquinone, $1 \mu \mathrm{M}$ indigocarmine, $10 \mu \mathrm{M}$ anthraquinone-2-sulfonate, and 5 $\mu \mathrm{M}$ benzyl viologen. After equilibration at each potential, the optical spectrum was recorded, and the course of the reduction of heme was followed by the increase in the $\alpha$-band absorption at $559 \mathrm{~nm}$ relative to a $575 \mathrm{~nm}$ reference wavelength. The absorption difference was plotted against potential, and the data were fit to single Nernst equation with $n=1$ (fixed).

Synthesis of 11-Mercaptoundecanoic Acid. 11-Mercaptoundecanoic acid (MUA) was synthesized and purified according to established procedures. ${ }^{34}$

Substrate Preparation. Glass slides, $1 \mathrm{~mm}$ in thickness, were sonicated first in detergent solution and then in water. This was followed by immersion in freshly prepared solution of 4/1 (v/ v) $95-98 \%$ sulfuric acid/30\% hydrogen peroxide (CAUTION: This solution is highly oxidizing and should be handled with extreme care) at room temperature for about $1 \mathrm{~h}$. The slides were then thoroughly rinsed with water, stripped of visible surface water with a stream of argon, and immediately placed into a high vacuum evaporator (Denton, Cherry Hill, NJ). Titanium was first deposited onto the glass by thermal evaporation under a vacuum of $6 \times 10^{-7}$ Torrs at a rate of $2 \AA / \mathrm{s}$ until a thickness of $300 \AA$ was reached. Then gold was deposited
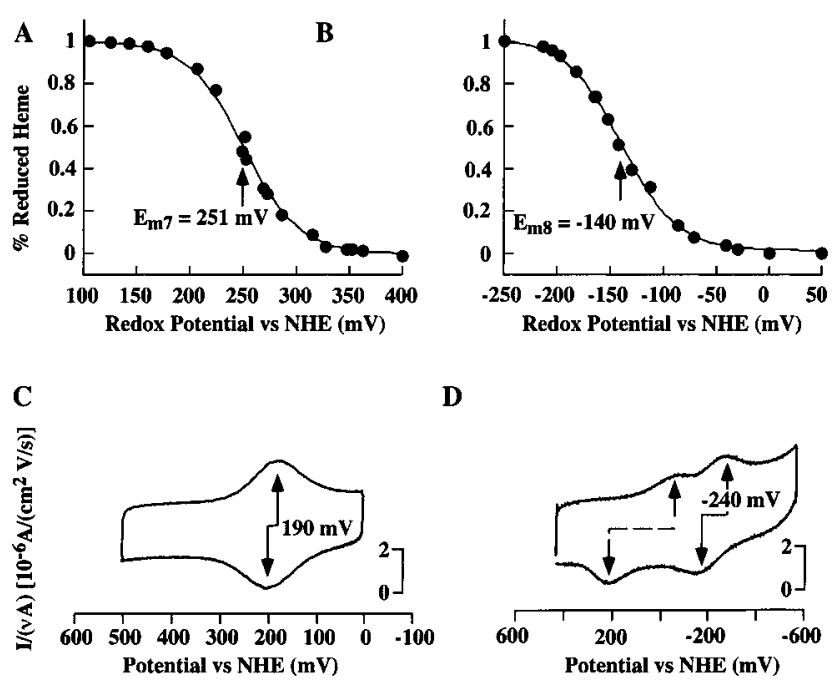

D

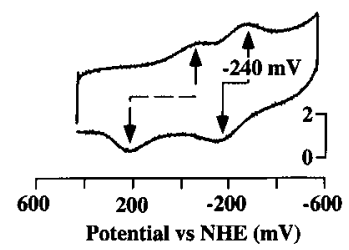

Figure 2. Comparison of the redox properties of cyt $c$ and the cyt $b$ maquette. Part A: Redox titration of cyt c performed at $\mathrm{pH} 7$ and 22 ${ }^{\circ} \mathrm{C}$ in $50 \mathrm{mM}$ Tris, $100 \mathrm{mM} \mathrm{NaCl}$. Part B: Redox titration of the cyt $b$ maquette performed at $\mathrm{pH} 8$ and $22{ }^{\circ} \mathrm{C}$ in $60 \mathrm{mM} \mathrm{NaCl}, 5 \mathrm{mM}$ phosphate. Part C: Cyclic voltammogram of cyt $c$ adsorbed on an MUA/Au electrode. Scan rate: $0.2 \mathrm{~V} / \mathrm{s}$. Solution condition: $10 \mathrm{mM}$ $\mathrm{NaCl}, 1 \mathrm{mM}$ sodium phosphate, $\mathrm{pH} 7.0,20^{\circ} \mathrm{C}$. Part D: Cyclic voltammogram of the cyt $b$ maquette adsorbed on MUA/Au electrodes. Scan rate: $10 \mathrm{~V} / \mathrm{s}$. Solution condition: $10 \mathrm{mM} \mathrm{NaCl}, 1 \mathrm{mM}$ sodium phosphate, $\mathrm{pH} 8.0,20^{\circ} \mathrm{C}$.

onto the glass by thermal evaporation under a vacuum of $6 \times$ $10^{-7}$ Torrs at a rate of $1 \AA / \mathrm{s}$ until a thickness of $1500 \AA$ was reached.

Freshly prepared gold electrodes were immersed in an absolute ethanol solution of MUA (approximately $1 \mathrm{mM}$ ) for more than $10 \mathrm{~h}$ to allow near complete self-assembly. Before protein adsorption, the MUA coated gold electrodes were washed first with absolute ethanol and then water. The MUA coated electrodes were then submerged for 30-60 min in solutions of approximately $0.01 \mathrm{mM}$ protein in $10 \mathrm{mM}$ ionic strength phosphate buffer, $\mathrm{pH} 7.0$ for cyt $c$, and $\mathrm{pH} 8.0$ for cyt $b$ maquette.

Cyclic Voltammetry. The reference electrode, the counter electrode and the working electrode were respectively $\mathrm{Ag} / \mathrm{AgCl}$ in $\mathrm{NaCl}(3 \mathrm{M})$, a platinum foil and the gold slide coated with proteins. The supporting electrolyte solutions were $10 \mathrm{mM} \mathrm{NaCl}$ and $1 \mathrm{mM}$ sodium phosphate. The geometric area of the working electrode was typically $0.5 \mathrm{~cm}^{2}$. The measured potentials are all converted relative to the normal hydrogen electrode (NHE) $\left(\mathrm{Ag} / \mathrm{AgCl}\right.$ in $3 \mathrm{M} \mathrm{NaCl}+0.196 \mathrm{~V}$ at $\left.25^{\circ} \mathrm{C}\right)$. Cyclic voltammograms were recorded on a workstation BAS 100B (Bioanalytical Systems Inc., W. Lafayette, IN) at a resolution of 1000 data points/V. The potential scan rates $(v)$ ranged from 0.01 to $297 \mathrm{~V} / \mathrm{s}$. The acquisition of the data was performed without electronic filtering for $v$ higher than $1 \mathrm{~V} / \mathrm{s}$. The values of the internal resistance of the solution were compensated manually.

\section{Results}

Cyclic Voltammetry of Heme Proteins on MUA/Gold in Comparison to the Classical Redox Potentiometry in Aqueous Solutions. Figure 2 emphasizes the qualitative similarity of the designed heme protein maquette with respect to the redox properties of natural proteins, typified by cyt $c$, which is used here for comparison. Panels A and B of Figure 2 show typical redox titrations obtained for cyt $c$ and the cyt $b$ maquette, respectively. Both titrations fit to a single $n=1$ Nernst curve, 
with an $E_{\mathrm{m} 7}$ value for cyt $c$ at $251 \mathrm{mV}$ and an $E_{\mathrm{m} 8}$ value for the cyt $b$ maquette at $-140 \mathrm{mV}$.

Panels C and D of Figure 2 show cyclic voltammograms obtained for the same proteins adsorbed on MUA/Au electrode. The peaks in Figure 2C are due to the one-electron oxidation and reduction of adsorbed cyt $c$. We estimate the surface coverage of electroactive cyt $c$ to be $8 \times 10^{-12} \mathrm{~mol} / \mathrm{cm}^{2}$ by integration of the charge under the anodic peak. This corresponds to $2100 \AA^{2} /$ molecule. For a reversible couple, the surface formal potential, defined as the average of the anodic peak potential $\left(E_{\mathrm{p}, \mathrm{a}}\right)$ and the cathodic peak potential $\left(E_{\mathrm{p}, \mathrm{c}}\right)$, corresponds to the biochemist's redox midpoint potential $\left(E_{\mathrm{m}}\right)$. For cyt $c$ associated with the MUA monolayer, this was $+0.19 \mathrm{~V}$ versus NHE, $0.06 \mathrm{~V}$ more negative than the $E_{\mathrm{m}}$ value determined in solution. Such downward shifts can be ascribed to the negatively charged surface on which the protein is adsorbed. The sign and extend of the shift agree well with $E_{\mathrm{m}}$ shifts for cyt $c$ bound to mitochondrial membranes ${ }^{35,36}$ and to photosynthetic reaction centers. ${ }^{37}$

Figure 2D shows a cyclic voltammogram obtained for the cyt $b$ maquette adsorbed on MUA/Au electrodes. There are two cathodic and two anodic peaks in the voltammogram. The higher potential peaks are also present in the cyclic voltammogram for MUA/Au electrodes without adsorbed heme protein maquettes, and correspond to the protonation and deprotonation of the MUA carboxylic acid group. These peaks do not appear in the cyclic voltammograms obtained for cyt $c$ in Figure 2C because the cathodic potential scan in Figure $2 \mathrm{C}$ was not extended to the negative potentials that induce the protonation of the MUA carboxylic acid group.

Similar protonation and deprotonation waves of the surface carboxylic acid groups have been found in the voltammetric response of a silver electrode coated with a mixed molecular film of MUA and 1-decanethiol. ${ }^{38}$ The theory underlying the voltammetric peaks corresponding to the protonation and deprotonation of the MUA carboxylic acid group has been previously discussed in detail. ${ }^{39}$ According to this theory, at $\mathrm{pH}$ values far from the $\mathrm{p} K_{\mathrm{a}}$ of the carboxylic acid groups on the surface, the film is either virtually fully protonated or fully deprotonated at all potentials. For either condition, the voltammograms are expected to be featureless. At $\mathrm{pH}$ values close to the $\mathrm{p} K_{\mathrm{a}}$, the double layer capacitance varies in response to the electrostatic potential at the plane of carboxylic acid groups, reaching a maximum value when the film is half ionized, inducing voltammetric peaks in both cathodic and anodic scans.

The lower potential peaks in Figure $2 \mathrm{D}$ are due to the oxidation and reduction of adsorbed cyt $b$ maquettes. The heme protein maquette/MUA surface formal potential or $E_{\mathrm{m} 8}$ value is $-0.24 \mathrm{~V}$ versus NHE. This is about $0.1 \mathrm{~V}$ more negative than the solution $E_{\mathrm{m}}$ value, which is directionally the same and similar to that displayed by cyt $c$. The surface coverage of the electroactive hemes on the MUA/Au surface is estimated to be $5 \times 10^{-12} \mathrm{~mol} / \mathrm{cm}^{2}$ by integration of the charge under the anodic peak. Since the ratio of heme per cyt $b$ maquette was 0.7 , the estimated surface coverage corresponds to an area of $2300 \AA^{2} /$ molecule. This area is about $10 \%$ higher than the area per cyt c. The cross section area of the maquette is about $1200 \AA^{2}$ / molecule when the protein maquette is oriented with its helix axis parallel to the surface and about $600 \AA^{2} /$ molecule when the protein maquette is oriented with its helix axis perpendicular to the surface (which the surface change distribution should favor, Figure 1B). ${ }^{14,15}$ Thus regardless of how the molecules lie on the electrode (Figure 1B), the molecules did not form a close-packed monolayer.

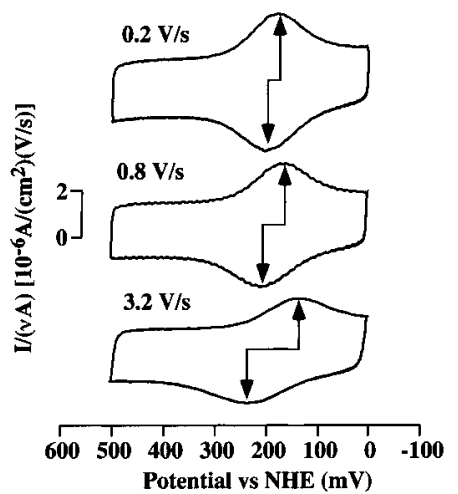

Figure 3. Cyclic voltammograms obtained for cyt $c$ adsorbed on an MUA/Au electrode acquired at different potential scan rate. From top to bottom: $v=0.2,0.8$, and $3.2 \mathrm{~V} / \mathrm{s}$. Solution condition: $10 \mathrm{mM} \mathrm{NaCl}$, $1 \mathrm{mM}$ sodium phosphate, $\mathrm{pH}$ 7.0. Temperature: $20^{\circ} \mathrm{C}$.

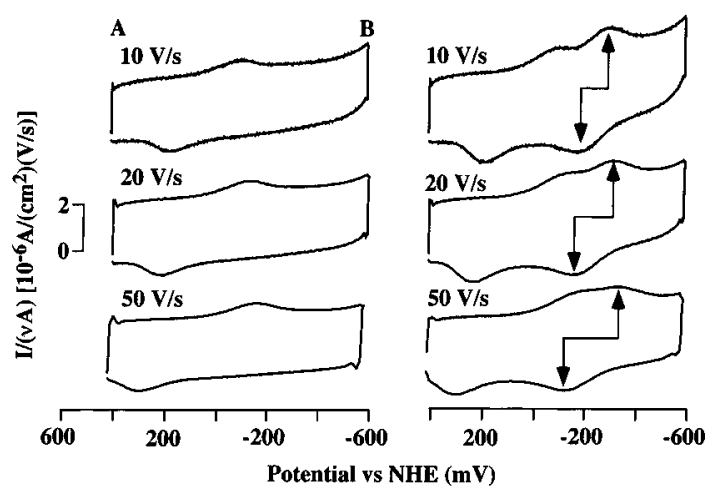

Figure 4. Cyclic voltammograms obtained for the cyt $b$ maquette adsorbed on an MUA/Au electrode acquired at different potential scan rate. Part A: Voltammograms of the MUA/Au electrode before protein adsorption. Part B: After protein adsorption. From top to bottom: $v=10,20$, and $50 \mathrm{~V} / \mathrm{s}$. Solution condition: $10 \mathrm{mM} \mathrm{NaCl}, 1 \mathrm{mM}$ sodium phosphate, $\mathrm{pH}$ 8.0. Temperature: $20^{\circ} \mathrm{C}$.

Electron-Transfer Rates of Heme Proteins on MUA/Gold. To obtain the electron-transfer rates between the electrode and the hemes of the cyt $c$ and cytocrome $b$ maquette immobilized on the MUA/Au electrode, cyclic voltammograms were acquired over a range of voltammetric scan rates. Figure 3 shows cyclic voltammograms obtained for cyt $c$ and Figure 4 for cyt $b$ maquette. Note in Figure 4 that the peak-to-peak separations corresponding to the protonation and deprotonation of the carboxylic acid surface also increase with scan rate. The scan rate dependence of the peak-to-peak separations in the cyclic voltammograms can be explained by the electron transfer kinetic theory for immobilized electrode reactants based on the Marcus theory. Since this theory was previously discussed in detail, ${ }^{25}$ we will describe it only briefly.

For a simple, reversible ET reaction between redox centers bound to a electrode, the dependency of the reduction rate constant $k_{\mathrm{red}, \eta}$ and the oxidation rate constant $k_{\mathrm{ox}, \eta}$ on the overpotential (free energy) $\eta=E-E^{\circ}$ can be expressed by the Butler-Volmer equations:

$$
\begin{gathered}
k_{\mathrm{red}, \eta}=k^{0} \exp \left(-\frac{\eta}{2 k_{\mathrm{B}} T}\right) \\
k_{\mathrm{ox}, \eta}=k^{0} \exp \left(\frac{\eta}{2 k_{\mathrm{B}} T}\right)
\end{gathered}
$$

where $k^{0}$ is the standard rate constant at free energy equal to zero. 


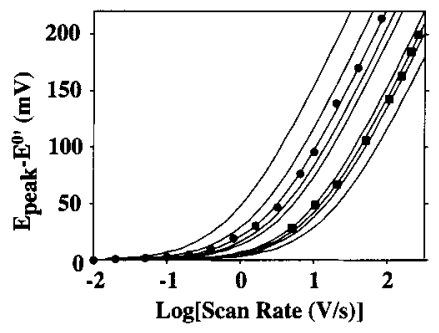

Figure 5. Half values of peak-to-peak separations $\left(E_{\text {peak }}-E^{\circ}{ }^{\prime}\right)$ as function of $\log$ scan rate. Solid circles: measured half values of peakto-peak separation obtained from voltammograms of cyt $c$ adsorbed on an MUA/Au electrode. Solid squares: measured half values of peakto-peak separation obtained from voltammograms of the cyt $b$ maquette adsorbed on an MUA/Au electrode. Lines: calculated $\left(E_{\text {peak }}-E^{\circ}\right)$ as a function of $\log (v)$ at different $k^{0}$ values. The calculated curves were generated by simulating voltammograms at different $k^{0}$ and $v$ values, following eqs $3-6$. Reorganization energy $\lambda$ is assumed to be $1 \mathrm{eV}$. From top to bottom: $k^{0}=10,20,30,40,50,100,120,140$, and $200 \mathrm{~s}^{-1}$.

The Butler-Volmer formulation predicts a continuous, exponential increase of the rate of ET with $(-\eta)$. The Marcus relations predicts also that the rate of ET increases with $(-\eta)$ when $(-\eta) / \lambda \ll 1$ ( $\lambda$ is the reorganization energy) but reaches maximum at $(-\eta)=\lambda$ and even decreases at larger $(-\eta)$ (the inverted region). According to Chidsey, ${ }^{40}$ for ET involving a metal interface, electronic distribution about the Fermi level has to be taken into account, and the Marcus relations can be expressed as follows:

$$
\begin{aligned}
& k_{\mathrm{red}, \eta}=\mu \rho k_{\mathrm{B}} T \int_{-\infty}^{\infty} \frac{\exp \left\{-\left(x-(\lambda+\eta) / k_{\mathrm{B}} T\right)^{2}\left(k_{\mathrm{B}} T / 4 \lambda\right)\right\}}{1+\exp (x)} \mathrm{d} x \\
& k_{\mathrm{ox}, \eta}=\mu \rho k_{\mathrm{B}} T \int_{-\infty}^{\infty} \frac{\exp \left\{-\left(x-(\lambda-\eta) / k_{\mathrm{B}} T\right)^{2}\left(k_{\mathrm{B}} T / 4 \lambda\right)\right\}}{1+\exp (x)} \mathrm{d} x
\end{aligned}
$$

where $x$ is the electron energy relative to the Fermi level, $\mu$ is the distance dependent electronic coupling between electrode and redox centers, and $\rho$ is the density of electronic states in the metal electrode. The standard rate constant at zero free energy, $k^{0}$, is then given by eqs 3 or 4 using $\eta=0$.

The calculations of linear sweep voltammetry were performed using eqs $1-2$ or $3-4$ for $k_{\mathrm{red}, \eta}$ and $k_{\mathrm{ox}, \eta}$ and the following iterative equations for the instantaneous current of first-order reaction $i_{\mathrm{F}, \eta}$ and the instantaneous fraction of reduced redox centers $P_{\text {red, } \eta}$ at each potential step $\eta$

$$
\begin{gathered}
i_{F, \eta}=n F \Gamma A\left(k_{\mathrm{ox}, \eta} P_{\mathrm{red}, \eta}-k_{\mathrm{red}, \eta}\left(1-P_{\mathrm{red}, \eta}\right)\right) \\
P_{\text {red }, \eta}=P_{\text {red }, \eta-\delta \eta}-\frac{i_{F, \eta-\delta \eta}}{n F \Gamma A \nu} \delta \eta
\end{gathered}
$$

where $n$ is the number of electrons participating in electrontransfer per redox center, $\Gamma$ is the surface coverage of the redox center, $A$ is the electrode area, $v$ is the potential scan rate, and $\delta \eta$ is the small increment of the potential.

Peak-to-peak separations were calculated by simulating voltammetric waves at different $k^{0}$ and $v$ values, following eqs 3-6. In Figure 5, half values of peak-to-peak separations were plotted as a function of log scan rate and compared to the measured data. Within the scan rates supported by the instrument, peak-to-peak separations are relatively insensitive to the value of reorganization energy, which therefore cannot be determined with any certainty. For the calculations of Figure 5 , we have assumed an arbitrary value of $1 \mathrm{eV}$, a reasonable estimate of $\lambda$ for this reaction. Comparison of the data to the calculated curves revealed the apparent rate constant at zero free energy $\left(k^{0}\right)$ of electron transfer between the cyt $c$ heme and the electrode to be $30 \mathrm{~s}^{-1}$. Similarly, $k^{0}$ of electron transfer between the heme in cyt $b$ maquette and the electrode was found to be $120 \mathrm{~s}^{-1}$.

Proton-Coupled Electron Transfer of Cytochrome $b$ Maquettes. Proton coupling to cofactor oxidation/reduction requires that an acid/base group(s) on the cofactor or nearby shift(s) its $\mathrm{p} K$ value depending on the oxidation state of a redox cofactor. Oxidizing the cofactor increases its positive charge, which thereby reduces the proton affinity of the acid/base group(s). Hence, the $\mathrm{p} K$ of an acid/base group is generally lower when the cofactor is in the oxidized state. It follows that the redox midpoint potential $E_{\mathrm{m}}$ value of the cofactor will in turn depend on the protonation state of the acid/base group, decreasing as the $\mathrm{pH}$ increases.

By assuming that there is only one acid/base group coupled to the heme oxidation/reduction, a simple relationship can be found to relate redox midpoint potential and $\mathrm{pH}$. The following equilibrium is set up:

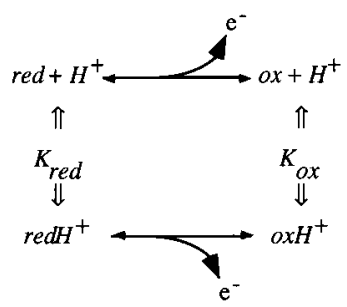

where red represents the protein state where the heme is reduced and the coupled acid/base group is unprotonated, ox represents the protein state where the heme is oxidized and the coupled $\mathrm{acid} / \mathrm{base}$ group is unprotonated, $\mathrm{redH}^{+}$represents the protein state where the heme is reduced and the coupled acid/base group is protonated, and $\mathrm{oxH}^{+}$represents the protein state where the heme is oxidized and the coupled acid/base group is protonated. $K_{\text {red }}$ is the proton binding affinity in the reduced state, and $K_{\text {ox }}$ is the proton binding affinity in the oxidized state.

The total amount of protein in reduced state is

$$
\begin{aligned}
{\left[\operatorname{red}_{\mathrm{tot}}\right]=} & {\left[\mathrm{redH}^{+}\right]+[\mathrm{red}]=} \\
& {\left[\mathrm{redH}^{+}\right]\left(1+\frac{[\mathrm{red}]}{\left[\mathrm{redH}{ }^{+}\right]}\right)=\left[\operatorname{redH}^{+}\right]\left(1+\frac{K_{\mathrm{red}}}{\left[H^{+}\right]}\right) }
\end{aligned}
$$

Similarly, the total amount of protein in oxidized state is

$$
\begin{aligned}
& {\left[\mathrm{ox}_{\mathrm{tot}}\right]=\left[\mathrm{oxH}^{+}\right]+[\mathrm{ox}]=} \\
& \quad\left[\mathrm{oxH}^{+}\right]\left(1+\frac{[\mathrm{ox}]}{\left[\mathrm{oxH}^{+}\right]}\right)=\left[\mathrm{oxH}^{+}\right]\left(1+\frac{K_{\mathrm{ox}}}{\left[\mathrm{H}^{+}\right]}\right)
\end{aligned}
$$

At any potential, the Nernst equation dictates that

$$
E_{\mathrm{m}}+k_{\mathrm{B}} T \ln \left(\frac{\left[\mathrm{ox}_{\mathrm{tot}}\right]}{\left[\mathrm{red}_{\mathrm{tot}}\right]}\right)=E_{0}+k_{\mathrm{B}} T \ln \left(\frac{\left[\mathrm{oxH}^{+}\right]}{\left[\mathrm{redH}^{+}\right]}\right)
$$

where $E_{0}$ is the redox midpoint potential of the fully protonated protein. Thus 


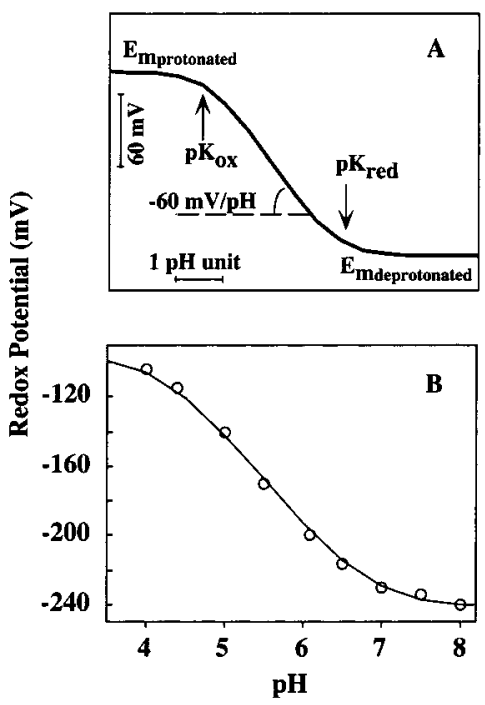

Figure 6. $\mathrm{pH}$ dependency of redox midpoint potentials. Part A: Theoretical curve corresponding to cofactor oxidation/reduction coupled to protonation of a single acid/base group. The slope in the linear portion of the $E_{\mathrm{m}}$ curve reaches approximately $-0.06 \mathrm{~V} / \mathrm{pH}$ unit in the case when $\mathrm{p} K_{\mathrm{ox}}$ and $\mathrm{p} K_{\text {red }}$ are well separated. Part B: $\mathrm{pH}$ dependency of redox midpoint potentials obtained from cyclic voltammograms of the cyt $b$ maquette adsorbed on an MUA/Au electrode. Circles: Experimental data. Line: The experimental data were fitted using eq 10 with $\mathrm{p} K_{\text {red }}=6.8, \mathrm{p} K_{\mathrm{ox}}=4.25$, and $E_{0}=-0.095 \mathrm{~V}$.

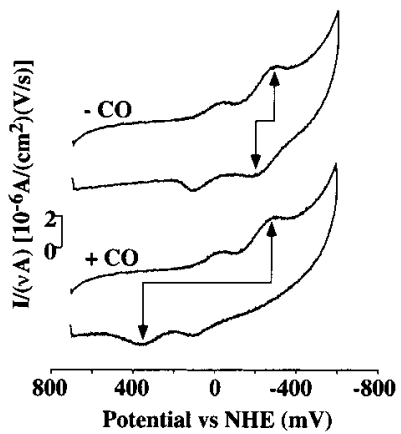

Figure 7. Effect of $\mathrm{CO}$ on the cyclic voltammograms for the cyt $b$ maquette adsorbed on an MUA/Au electrode. These cyclic voltammograms are obtained for the cyt $b$ maquette adsorbed on an MUA/Au electrode in argon-saturated (top curve) and CO-saturated (bottom curve) electrolyte solution. The arrows indicate the voltammetric peaks assigned to the heme reduction/oxidation. The other peaks are associated with protonation/deprotonation of the MUA headgroup. Scan rate: 2 $\mathrm{V} / \mathrm{s}$. Solution condition: $10 \mathrm{mM} \mathrm{NaCl}, 1 \mathrm{mM}$ sodium phosphate, $\mathrm{pH}$ 8.0. Temperature: $20^{\circ} \mathrm{C}$.

$$
\begin{aligned}
E_{\mathrm{m}}=E_{0}+k_{\mathrm{B}} T \ln \left(\frac{\left[\mathrm{oxH}^{+}\right]\left[\mathrm{red}_{\mathrm{tot}}\right]}{\left[\mathrm{redH}^{+}\right]\left[\mathrm{ox}_{\mathrm{tot}}\right]}\right)= \\
E_{0}+k_{\mathrm{B}} T \ln \left(\frac{1+K_{\mathrm{red}} /\left[\mathrm{H}^{+}\right]}{1+K_{\mathrm{ox}} /\left[\mathrm{H}^{+}\right]}\right)= \\
E_{0}+k_{\mathrm{B}} T \ln \left(\frac{K_{\mathrm{red}}+\left[\mathrm{H}^{+}\right]}{K_{\mathrm{ox}}+\left[\mathrm{H}^{+}\right]}\right)
\end{aligned}
$$

Figure 6A shows the theoretical curve corresponding to cofactor oxidation/reduction coupled to protonation of a single acid/base group. The slope in the linear portion of the $E_{\mathrm{m}} / \mathrm{pH}$ curve reaches approximately $-0.06 \mathrm{~V} / \mathrm{pH}$ unit in the case when $\mathrm{p} K_{\mathrm{ox}}$ and $\mathrm{p} K_{\text {red }}$ $\left(\mathrm{p} K_{\mathrm{a}}=\log \left(1 / K_{\mathrm{a}}\right)\right)$ are well separated.

Figure $6 \mathrm{~B}$ plots the $\mathrm{pH}$ dependency of redox midpoint potentials obtained from cyclic voltammograms of cyt $b$ maquette adsorbed on an MUA/Au electrode. $E_{\mathrm{m}}$ values decrease from $\mathrm{pH} 4\left(E_{\mathrm{m} 4}=-0.11 \mathrm{~V}\right)$ to $\mathrm{pH} 8\left(E_{\mathrm{m} 8}=-0.24 \mathrm{~V}\right)$. The experimental data were fitted using eq 10 with $\mathrm{p} K_{\text {red }}=6.8$, $\mathrm{p} K_{\mathrm{ox}}=4.25$, and $E_{0}=-0.095 \mathrm{~V}$.

Redox-Coupled CO Binding/Release of Cytochrome $b$ Maquettes. Figure 7 shows the cyclic voltammograms for cyt $b$ maquette adsorbed on an MUA/Au electrode, both in the presence and absence of $\mathrm{CO}$. In the presence of $\mathrm{CO}$, the reduction wave in the cathodic potential scan is identical to that without $\mathrm{CO}$, while the oxidation wave in the anodic potential scan occurs at much higher potential compared to that without $\mathrm{CO}$. In concert with spectroscopic studies of $\mathrm{CO}$ interactions with maquette monolayers, ${ }^{16}$ the voltammogram in the presence of $\mathrm{CO}$ indicates that upon reduction of the heme, $\mathrm{CO}$ becomes ligated to the ferrous bis-histidyl heme in the maquettes by displacing one of the histidines. This occurs before the anodic scan. In the anodic potential scan, the CO-bound heme was oxidized at $0.35 \mathrm{~V}$ versus NHE, instead of $-0.2 \mathrm{~V}$ expected for the bis-histidyl hemes in the maquette. $\mathrm{CO}$ was released upon the oxidation of the heme and the heme returned to the bis-histidyl ligation state. Thus, in the subsequent cathodic potential scan, the reduction wave repeats the first cycle (without $\mathrm{CO}$ ). This reduction $-\mathrm{CO}$ binding-oxidation- $\mathrm{CO}$ release cycle is illustrated as follows:

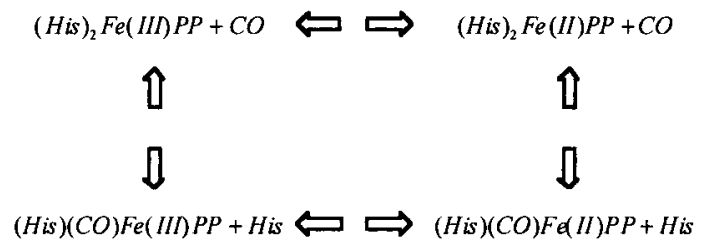

where Fe(III)PP and Fe(II)PP denote the ferric and ferrous heme, respectively. The cyclic voltammetric scans proceed in the clockwise direction of the above cycle. Peak positions in the presence of $\mathrm{CO}$ were not dramatically sensitive to increases and decreases of scan rate.

The $\mathrm{CV}$ response to the $\mathrm{CO}$ binding/release and ligand exchange indicates a major difference in dynamics with respect to the oxidation/reduction coupled proton exchange. In contrast to the proton exchange that has not been found rate limiting on the time scale of electron tunneling between the electrode and the heme, dissociation of $\mathrm{CO}$ from the reduced heme and reassociation with the oxidized heme appear slower than electron transfer.

\section{Discussion}

There are several advantages of using $\mathrm{COO}^{-}$terminated alkanethiol as a linker for immobilizing heme protein maquettes on gold surfaces. First, the structural conservation of proteins on transfer from solution to surfaces is critical in the study of protein electron-transfer properties in monolayer films, and the $\mathrm{COO}^{-}$surface presents a hydrophilic interface which better conserves the solution structure of the proteins as compared to a nonpolar surface. Second, the negatively charged $\mathrm{COO}^{-}$terminated surface provides a means to bind and orient the proteins through electrostatic interactions. By designing the charged residue patches, we were able to enhance the interaction of the maquette with the electrode surface, enabling further investigation of electron transfer, proton-coupled electron transfer, and redox-coupled $\mathrm{CO}$ binding and release.

The formation of monolayers of cyt $c$ and the cyt $b$ maquette at the surface of MUA/Au proceeds predominantly by electrostatic interaction. The density of the proteins in the films 
decreased substantially when the films were incubated in a buffer with ionic strength higher than $100 \mathrm{mM}$.

In the electron-transfer reactions between the gold surface and the hemes of cyt $c$ or cyt $b$ maquette, the electrons tunnel through the MUA alkane spacer and the protein medium. The electron-transfer rates between electrode and the heme $\left(k_{\mathrm{et}}\right)$ provide additional information on the geometry of the protein at the surface by providing an estimate of the electron tunneling distance between the hemes and the electrode. The free energy optimized electron tunneling rate falls off approximately exponentially with the tunneling distance, more or less steeply depending upon the effective barrier height of the insulating medium. In intraprotein electron transfer, the tunneling rate can be estimated from ${ }^{5}$

$$
\begin{array}{r}
\log k_{\mathrm{et}}=13.0-\left(\beta_{\text {spacer }} R_{\text {spacer }}+\beta_{\text {protein }} R_{\text {protein }}\right) / 2.303- \\
3.1(\Delta G+\lambda)^{2} / \lambda
\end{array}
$$

where $R_{\text {spacer }}$ is the thickness of the MUA spacer and $R_{\text {protein }}$ is the distance between the edge of the heme and the plane of $\mathrm{COO}^{-}$groups of the MUA monolayer. Numerous studies suggest that the MUA monolayer assembly have fully extended all-trans alkyl chains packed at $30^{\circ}$ angle relative to the surface normal, ${ }^{41}$ thus $R_{\text {spacer }}$ is estimated to be $12.3 \AA$. The $\beta$ value of electron tunneling through the alkane spacer, $\beta_{\text {spacer, }}$, is $1.0 \AA^{-1}$ on the basis of experimental examination of tunneling in alkanethiol monolayers. ${ }^{37-40}$ And the average $\beta$ value of electron

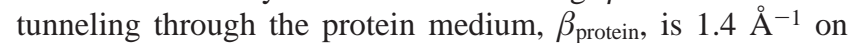
the basis of experimental examination of tunneling in proteins. ${ }^{2-5}$

The free energy optimized rate, and hence the estimated tunneling distance, will depend on the choice of the reorganization energy $(\lambda)$. The $\lambda$-dependent term can take a classical Marcus form (as assumed in eqs 3 and 4) or a quantized form (as in eq 11). For a given electron-transfer rate at zero driving force $\left(k^{0}\right)$, both the classical and quantum expressions, when integrated over the Fermi density of states in the electrode, generate similar CV peak separations as a function of scan rate for modest peak separations of less than $200 \mathrm{mV}$ (Figure 5). In both cases, $k^{0}$ is relatively insensitive to $\lambda$ over this range.

For cyt $c$, a $k^{0}$ of $30 \mathrm{~s}^{-1}$ yields a classical distance from heme

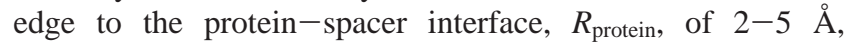
assuming reorganization energies of $0.5-1 \mathrm{eV}$. The quantum expressions yield a distance of 3-6 $\AA$ for the same reorganization energy range. A distance range of $4 \pm 2 \AA$ is consistent with the geometry where the heme pocket of cyt $c$, whose surroundings are positively charged due to the basic amino acids, is facing down and close to the plane of $\mathrm{COO}^{-}$groups of the MUA monolayer.

For the cyt $b$ maquette, similar distance estimates are about $3 \pm 2 \AA$. By taking into account the $R_{\text {spacer }}$, the total heme to electrode distance is calculated to be about $15 \AA$. The small $R_{\text {protein }}$ suggests that the heme in the cyt $b$ maquette is almost in contact with the surface of the $\mathrm{COO}^{-}$terminated alkanethiol layer. Since the measured $k_{\mathrm{et}}$ is near the physical limit of electron tunneling through the thickness of the alkanethiol monolayer, electron transfer appears to be tunneling limited and not restricted by a relatively slow proton transfer. Additional evidence that the electron transfer is not proton limited is provided by the experimental insensitivity of the peak separation versus scan rate behavior to changes in $\mathrm{pH}$.

According to the work of Shifman and co-workers ${ }^{1}$, the $\mathrm{pH}$ dependency of redox midpoint potential of the heme protein maquette at low $\mathrm{pH}$ region is due to the glutamates in the maquette. It is suggested that several glutamates are recruited into the four- $\alpha$-helix-bundle interior as a part of structural rearrangement on heme binding. Similar $\mathrm{p} K_{\mathrm{a}}$ 's are found here, close to 7 for reduced heme and 4 for oxidized heme. Although relatively little is presently known about the proton translocation in natural proteins, in a few systems certain carboxylate residues with unusually high $\mathrm{p} K$ values were proven to be part of protontransfer pathway, such as ferredoxin I, ${ }^{22}$ bacteriorhodopsin, ${ }^{46}$ photosynthetic reaction center, ${ }^{47}$ and cytochrome $c$ oxidase. ${ }^{48}$

The films of cyt $b$ maquettes after reduction, were able to bind $\mathrm{CO}$ reversibly. Furthermore, the binding and release of $\mathrm{CO}$ can be regulated by the applied potential on the electrode. This demonstrates that films of heme protein maquettes are sensitive to molecules dissolved in aqueous solution and can be of utility as chemical and biochemical sensors.

Acknowledgment. This research was supported by grant from NIH GM41048 and in part a MRSEC/IRG grant from NSF (DMR96-20668).

\section{References and Notes}

(1) Shifman, J. M.; Moser, C. C.; Kalsbeck, W. A.; Bocian, D. F.; Dutton, P. L. Biochemistry 1998, 37, 16815-16827.

(2) Bendall, D. S. Protein Electron Transfer; BIOS Scientific Publishers Ltd.: Oxford, 1996.

(3) Gray, H. B.; Winkler, J. R. Annu. Rev. Biochem. 1996, 35, 53761 .

(4) Moser, C. C.; Keske, J. M.; Warncke, K.; Farid, R. S.; Dutton, P. L. Nature 1992, 355, 796-802.

(5) Page, C. C.; Moser, C. C.; Chen, X.; Dutton, P. L. Nature 1999, $402,47-52$.

(6) Bryson, J. W.; Betz, S. F.; Lu, H. S.; Suich, D. J.; Zhou, H. X.; O’Neil, K. T.; DeGrado, W. F. Science 1995, 270, 935-41.

(7) DeGrado, W. F.; Wasserman, Z. R.; Lear, J. D. Science 1989, 243 , 622-628.

(8) Tuchscherer, G.; Scheibler, L.; Dumy, P.; Mutter, M. Biopolymers 1998, $47,63-73$.

(9) Robertson, D. E.; Farid, R. S.; Moser, C. C.; Urbauer, J. L.; Mulholland, S. E.; Pidikiti, R.; Lear, J. D.; Wand, A. J.; DeGrado, W. F.; Dutton, P. L. Nature 1994, 368, 425-32.

(10) Choma, C. T.; Lear, J. D.; Nelson, M. J.; Dutton, P. L.; Robertson, D. E.; DeGrado, W. F. J. Am. Chem. Soc. 1994, 116, 856-865.

(11) Gibney, B. R.; Rabanal, F.; Reddy, K. S.; Dutton, P. L. Biochemistry 1998, 37, 4635-4643.

(12) Rabanal, F.; DeGrado, W. F.; Dutton, P. L. J. Am. Chem. Soc. Commun. 1996, 118, 473-474.

(13) Gibney, B. R.; Dutton, P. L. De Novo Design and Synthesis of Heme Proteins. In Advances in Inorganic Chemistry; 2001; Academic Press: San Diego, Vol. 51, pp 409-455.

(14) Chen, X.; Moser, C. C.; Pilloud, D. L.; Dutton, P. L. J. Phys. Chem

$B$ 1998, 102, 6425-6432.

(15) Chen, X. X.; Moser, C. C.; Pilloud, D. L.; Gibney, B. R.; Dutton,

P. L. J. Phys. Chem. B 1999, 103, 9029-9037.

(16) Pilloud, D. L.; Rabanal, F.; Gibney, B. R.; Farid, R. S.; Dutton, P. L.; Moser, C. C. J. Phys. Chem. B 1998, 102, 1926-1937.

(17) Armstrong, F. A.; Heering, H. A.; Hirst, J. Chem. Soc. Rev. 1997, $26,169-179$

(18) Whitesides, G. M.; Laibinis, P. E. Langmuir 1990, 6, 87-96 and references therein.

(19) Clark, R. A.; Bowden, E. F. Langmuir 1997, 13, 559-565.

(20) Feng, Z. Q.; Imabayashi, S.; Kakiuchi, T.; Niki, K. J. Chem. Soc., Faraday Trans. 1997, 93, 1367-1370.

(21) Tarlov, M. J.; Bowden, E. F. J. Am. Chem. Soc. 1991, 113, 18471849.

(22) Chen, K. S.; Hirst, J.; Camba, R.; Bonagura, C. A.; Stout, C. D.; Burgess, B. K.; Armstrong, F. A. Nature 2000, 405, 814-817.

(23) Hirst, J.; Duff, J. L. C.; Jameson, G. N. L.; Kemper, M. A.; Burgess, B. K.; Armstrong, F. A. J. Am. Chem. Soc. 1998, 120, 7085-7094.

(24) Shen, B. H.; Martin, L. L.; Butt, J. N.; Armstrong, F. A.; Stout, C. D.; Jensen, G. M.; Stephens, P. J.; Lamar, G. N.; Gorst, C. M.; Burgess, B. K. J. Biol. Chem. 1993, 268, 25928-25939.

(25) Tender, L.; Carter, M. T.; Murray, R. W. Anal. Chem. 1994, 66 , $3173-3181$

(26) Rich, P. R. Aust. J. Plant Physiol. 1995, 22, 479-486.

(27) Michel, H. Nature 1999, 402, 602-603.

(28) Wikstrom, M. Biochemistry 2000, 39, 3515-3519.

(29) Yoshikawa, S.; Choc, M. G.; O’Toole, M. C.; Caughey, W. S. J. Biol. Chem. 1977, 252, 5498-508. 
(30) Decatur, S. M.; Boxer, S. G. Biochem. Biophys. Res. Commun. 1995, $212,159-164$.

(31) O'Connor, D. B.; Goldbeck, R. A.; Hazzard, J. H.; Kliger, D. S.; Cusanovich, M. A. Biophys. J. 1993, 65, 1718-1726.

(32) Gibney, B. R.; Rabanal, F.; Skalicky, J. J.; Wand, A. J.; Dutton, P. L. J. Am. Chem. Soc. 1999, 121, 4952-4960.

(33) Dutton, P. L. Redox Potentiometry: Determination of Midpoint Potentials of Oxidation-Reduction Components in Biological ElectronTransfer Systems. In Methods in Enzymology, Biomembranes, Physical and Chemical Methods; 1978; Vol. VII, Part C, Chapter 25; pp 411-435.

(34) Bain, C. D.; Troughton, E. B.; Tao, Y. T.; Evall, J.; Whitesides, G. M.; Nuzzo, R. G. J. Am. Chem. Soc. 1989, 111, 321-335.

(35) Dutton, P. L.; Wilson, D. F.; Lee, C. P. Biochemistry 1970, 9 , $5077-5082$

(36) Vanderkooi, J.; Erecinska, M.; Chance, B. Arch. Biochem. Biophys. 1973, 157, 531-40.

(37) Moser, C. C.; Dutton, P. L. Biochim. Biophys. Acta 1992, 1101, $171-176$.
(38) White, H. S.; Peterson, J. D.; Cui, Q. Z.; Stevenson, K. J. J. Phys Chem. B 1998, 102, 2930-2934.

(39) Smith, C. P.; White, H. S. Langmuir 1993, 9, 1-3.

(40) Chidsey, C. E. D. Science 1991, 251, 919-922.

(41) Strong, L.; Whitesides, G. M. Langmuir 1988, 4, 546-558.

(42) Dubois, L. H.; Nuzzo, R. G. Annu. Rev. Phys. Chem. 1992, 43, 437-463 and references therein

(43) Pilloud, D. L.; Chen, X. X.; Dutton, P. L.; Moser, C. C. J. Phys. Chem. B 2000, 104, 2868-2877.

(44) Sachs, S. B.; Dudek, S. P.; Hsung, R. P.; Sita, L. R.; Smalley, J. F.; Newton, M. D.; Feldberg, S. W.; Chidsey, C. E. D. J. Am. Chem. Soc 1997, 119, 10563-10564.

(45) Smalley, J. F.; Feldberg, S. W.; Chidsey, C. E. D.; Linford, M. R.; Newton, M. D.; Liu, Y. P. J. Phys. Chem. 1995, 99, 13141-13149.

(46) Lanyi, J. K. Biophys. J. 1996, 71, 541-543.

(47) Takahashi, E.; Wraight, C. A. Biochemistry 1992, 31, 855-66.

(48) Hellwig, P.; Rost, B.; Kaiser, U.; Ostermeier, C.; Michel, H.; Mantele, W. Febs Lett. 1996, 385, 53-57. 